\title{
Solving Non-Fourier Heat Conduction-Radiation Problem, a Semi-Analytical Approach
}

\author{
Armin Emamifar* and Majid Goodarzi \\ University of Ayatollah Ozma Boroujerdi, Boroujerd, Iran, P.O.B. 167 \\ emamifar@abru.ac.ir, majid.goodarzi@abru.ac.ir
}

\begin{abstract}
Objectives: In this study, the thermal analysis of a thin film exposed to laser heating, was investigated using Cattaneo Vernotte non-Fourier heat conduction model. Methods/Statistical Analysis: Nonfourier equations were derived based on radiative heat loss and also variable thermal conductivity. Then Variational Iteration Method (VIM) and Reduced Differential Transformation Method (RDTM) were used to solve these equations. Findings: VIM is an efficient choice to find an approximate solution of nonlinear non-Fourier heat conduction and its accuracy is higher than RDTM. Considering the thermal conductivity vary with temperature creates a significant difference in the temperature profiles, which shows the importance of nonlinear analysis of the problem. In certain values of relaxation time, temperature variations take place and out of that certain domain, variations of relaxation time doesn't effect on temperature at all. Application/Improvements: There is a remarkable difference between the Fourier and non-Fourier solutions of heat conduction equations that demonstrate the importance of non-Fourier analysis of this type of equations.
\end{abstract}

Keywords: Conduction-Radiation, Laser Heating, Non-Fourier, Semi-Analytical, RDTM, VIM

\section{Introduction}

Nowadays, using of energetic laser rays has more applications in medicine, manufacturing and material processes. In fact, the ability of the laser in controlling of depth and width of the heating of the specified place and the controlling of the power of the irradiated beam, make using of these tools interesting in the industry and medical applications ${ }^{1}$. The lasers are powerful sources of heat generation and the produced heat in the certified body by them should be analyzed properly and carefully, especially when radiative heat loss has a significant role in such cases. In fact, studying of heat transfer in some cases like the processes of melting and freezing in the furnaces ${ }^{2}$, heat pipes and combustion chambers ${ }^{3,4}$ and laser heating ${ }^{5}$, should be done in the combined mode of conduction-radiation.

One of the cases that the classic law of Fourier heat conduction confronts failure is transferring high heat flux in short period of time. Laser heating is a good example of better understanding this process. Therefore, it is better to use non-Fourier models in the laser heating problems. First $^{6,7}$ developed a new macroscopic heat conduction model according to a Fourier approach. Since this model showed a wave-like behavior for heat conduction, they called it heat wave model or in honor of these scientists, it was known as $(\mathrm{C}-\mathrm{V})$ model.

In the recent years, using of non-Fourier equations for investigating laser heating is reported in a large number of studies. $\mathrm{In}^{8}$ used a numerical method for analyzing of heat wave propagation on a body under laser irradiation. $\operatorname{In}^{9}$ used the non-Fourier model for the problem of laser heating of a thin film and solved it analytically. $\mathrm{In}^{10}$ investigated parabolic and hyperbolic heat equation of skin under short and long periods of laser irradiation. Non-Fourier analysis of the thermal damage caused by laser heating was the subject of a studying done $\mathrm{e}^{11,12}$ analyzed the laser heating of living tissues by the non-Fourier model in a newer study.

${ }^{*}$ Author for correspondence 
Since in the present work, thermal conductivity is variable and the thermal radiation is also taken into account, we are facing a non-linear problem (with the high degree of nonlinearity). Therefore it is the semi-analytical methods which are built for this purpose. VIM which is used in this paper is one of the most flexible and most powerful of these tools that are used for solving of linear or nonlinear equations in a large number of investigations. This method was invented ${ }^{13}$ and then many researchers emphasized on its suitable converging ${ }^{14-19}$. Recently many applications of this method have been reported. $\operatorname{In}^{20}$ developed three new equations of forth order of Emdenfowler type by VIM. A solution of the transverse forced vibrations in a thermo-elastic thin beam by VIM was the subject of a work done $\mathrm{e}^{21,22}$ used VIM to present an analytic solution for particle breakage equation. $\mathrm{In}^{23}$ solved the problem of flap wise bending vibration of rotating tapered beams by VIM.

RDTM is another method which is used in this study. Although this method is not as famous as VIM, it has a lot of abilities in solving PDE problems. In $^{24}$ introduced RDTM to solve PDE problems. Then they solved both non-linear and linear wave-like equations by RDTM in another study ${ }^{25}$. Solving of two dimensional Volterra integral equations by RDTM was the subject of study done ${ }^{26}$. Recently RDTM is used to solve partial differentialalgebraic equations ${ }^{27}$.

Only a few authors have presented semi-analytical solutions for non-linear non-Fourier heat equations. Homotopy perturbation method was utilized ${ }^{28}$ to derive explicit solutions for a nonlinear Fourier heat transfer problem. In $^{29}$ solved the same problem by variational iteration method (VIM). In ${ }^{30}$ in another work used Differential Transformation Method (DTM) to investigate convective-radiative lumped systems with variable surface emissivity and specific heat. The last three papers, mentioned above, have been used semi-analytical techniques to solve ordinary differential equations and to the best of the author's knowledge, semi analytical methods have not been utilized to solve partial differential equations of Fourier heat conduction problems.

In this study, the temperature distribution in a thin film under laser irradiation is determined using a Fourier approach. Non-linear equations were derived due to variable thermal conductivity and also radiative heat loss. Derived equations were solved by VIM and RDTM. Utilizing semi-analytical techniques to solve nonlinear PDE of non-Fourier heat conductionradiation equation is the originality and novelty and of this paper.

\section{Mathematical Modeling}

Figure 1 indicates a simplified schematic of the problem. A film with the thickness of $L$ and with the initial temperature of $T_{0}$ is heated by laser heat flux from the left side and left and right boundaries are kept at $T_{0}$ and zero temperatures, respectively. Moreover, radiative heat loss is considered.

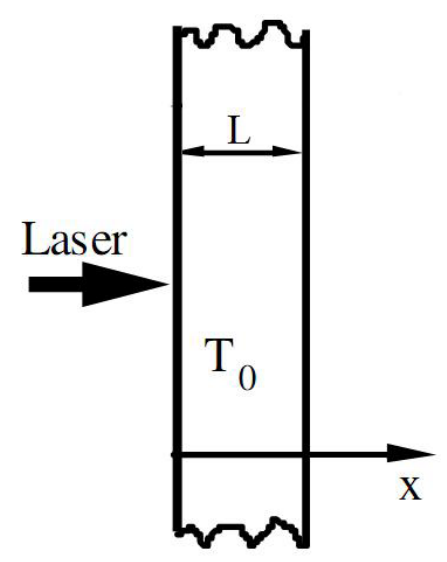

Figure 1. Schematic of the Problem.

Energy equation assuming the presence of a heat source is as follows:

$$
\rho c_{p} \frac{\partial T(x, t)}{\partial t}+\frac{\partial q_{C}(x, t)}{\partial x}+\frac{\partial q_{R}(x, t)}{\partial x}-g=0
$$

The conduction heat flux equation dominating on the concern problem based on the heat wave model $(6,7)$ is:

$$
\tau \frac{\partial q_{C}(x, t)}{\partial t}+q_{C}(x, t)+k \frac{\partial T(x, t)}{\partial x}=0
$$

The radiative heat flux can be used as follows ${ }^{31}$ :

$$
\frac{\partial q_{R}(x, t)}{\partial x}=\beta^{*}(1-\omega)\left(4 \pi \frac{\sigma T^{4}}{\pi}-G^{*}\right)
$$

A variation in thermal conductivity is considered as a function of the film temperature, as follows ${ }^{32}$ :

$$
k=k_{0}\left[1+\lambda\left(T(x, t)-T_{0}\right)\right]
$$

The heat flux of laser heat source is modeled as follows ${ }^{33,34}$ :

$$
g(x, t)=I(t)(1-R) \mu \exp (-\mu x)
$$


The following dimensionless parameters are introduced: $\tilde{t}=\frac{c_{0} t}{L}, \tilde{x}=\frac{x}{L}, \gamma=\lambda T_{0}, \quad V e=\frac{\tau c_{0}}{L}, \quad \mathrm{~N}=\frac{k_{0} c_{0}}{4 \alpha \sigma T_{0}^{3}}$, $\tilde{T}(\tilde{x}, \tilde{t})=\frac{T(x, t)}{T_{0}}, \tilde{q}(\tilde{x}, \tilde{t})=\frac{q_{C}(x, t) L}{T_{0} k_{0}}, G=\frac{G^{*}}{\sigma T_{0}^{3} / \pi}$, $\beta=\beta^{*} L(1-\omega)$.

Thus equations (1) and (2) are written the dimensionless form:

$$
\begin{gathered}
\frac{\partial \tilde{T}(\tilde{x}, \tilde{t})}{\partial \tilde{t}}+V e \frac{\partial \tilde{q}(\tilde{x}, \tilde{t})}{\partial \tilde{x}}+ \\
\frac{1}{N}\left(\beta \tilde{T}^{4}(\tilde{x}, \tilde{t})-\frac{G}{4}\right)-2 \psi_{0} \phi(\tilde{t}) \exp (-B \tilde{x})=0 \\
V e \frac{\partial \tilde{q}(\tilde{x}, \tilde{t})}{\partial \tilde{t}}+\tilde{q}(\tilde{x}, \tilde{t})+[1+\gamma \tilde{T}(\tilde{x}, \tilde{t})] \frac{\partial \tilde{T}(\tilde{x}, \tilde{t})}{\partial \tilde{x}}=0
\end{gathered}
$$

The dimensionless form of the initial and boundary are:

$$
\tilde{T}(\tilde{x}, 0)=0, \tilde{q}(\tilde{x}, 0)=0, \tilde{T}(0, \tilde{t})=1, \tilde{T}(1, \tilde{t})=0 .
$$

\section{Basic Concepts of VIM}

In this section, the fundamental ideas of VIM are presented. Consider the following nonlinear differential equation in its general form:

$$
L u(x, t)+N u(x, t)=g(x, \mathrm{t})
$$

Where $L$ and $\mathrm{N}$ are linear and nonlinear operators respectively and $g(x, t)$ is the non-homogeneous term, which in turn is an analytic continuous function. According to VIM, the correction functional can be defined as follows:

$$
\begin{aligned}
& u_{n+1}(x, t)=\mathrm{u}_{n}(x, t) \\
& +\int_{0}^{t} \lambda\left(L u_{n}(\mathrm{x}, \tau)+N \tilde{u}_{n}(\mathrm{x}, \tau)-g(\mathrm{x}, \tau)\right) d \tau
\end{aligned}
$$

Where, $\lambda$ is Lagrange multiplier that can be determined using various ways. The subscript of $\mathrm{n}$ represents the $\mathrm{n}^{\text {th }}$ approximate of the restricted variation, where $\delta \tilde{u}_{n}=0$. The one is free to choose the initial guess $\left(u_{0}\right)$, but it can be achieved by solving the following equation:

$$
L u_{0}(x, t)=0
$$

Finally, the converged solution that is the exact solution of the problem can be obtained by the successive limit of approximations:

$$
u_{n+1}(x, t)=u_{n}(x, t) \quad \text { or } u(x, t)=\lim _{n \rightarrow \infty} u_{n}(x, t)
$$

\section{Basic Concepts of RDTM}

In this section, the basic ideas of RDTM are presented. Suppose that $u(x, t)$ is analytic and continuously differentiable with respect to $t$. Then:

$$
U_{k}(x)=\frac{1}{k !}\left[\frac{\partial^{k}}{\partial t^{k}} u(x, t)\right]_{t=0}
$$

Where $U_{k}(x)$ is transformed of $u(x, t)$. Then the inverse of $U_{k}(x)$ is as follows:

$$
u(x, t)=\sum_{k=0}^{\infty} U_{k}(x) t^{k}
$$

By combining (14) and (15):

$$
u(x, t)=\sum_{k=0}^{\infty} \frac{1}{k !}\left[\frac{\partial^{k}}{\partial t^{k}} u(x, t)\right]_{t=0} t^{k}
$$

Some functions and their transformation are presented in Table 1. Now, consider a general PDE as follows:

$$
L u(x, t)+R u(x, t)+N u(x, t)=g(x, t)
$$

Where $L$ is the highest order of differentiation which is invertible. $R$ is the linear differential operator which is containing of remaining terms, $\mathrm{Nu}$ is containing of nonlinear terms and $g$ is the non-homogeneous term. An initial condition is supposed as follows:

$$
u(x, 0)=f(x)
$$

Finally, with general equation of (17) and initial condition of (18) and also with transformed functions of Table 1, it can be achieved to an iterative formula. For the problem of this paper $L=\frac{\partial}{\partial t}$ and the iterative formula is
as follows:

$$
(k+1) U_{k+1}(x)=G_{k}(x)-R U_{k}(x)-N U_{k}(x)
$$

Table 1. Reduced Differential Transformation

\begin{tabular}{|l|l|}
\hline Functional Form & Transformed Form \\
\hline$u(x, t)$ & $U_{k}(x)=\frac{1}{k !}\left[\frac{\partial^{k}}{\partial t^{k}} u(x, t)\right]_{t=0}$ \\
\hline$w(x, t)=u(x, t) \mathrm{v}(x, t)$ & $W_{k}(x)=\sum_{r=0}^{k} U_{r}(x) V_{k-r}(x)$ \\
\hline
\end{tabular}




\begin{tabular}{|l|l|}
\hline$w(x, t)=\sin (\omega t+\alpha)$ & $W_{k}(x)=\frac{\omega^{k}}{k !} \sin \left(\frac{\pi k}{2 !}+\alpha\right)$ \\
\hline$w(x, t)=\frac{\partial^{r}}{\partial t^{r}} u(x, t)$ & $W_{k}(x)=(k+1)(k+2) \ldots$ \\
$(k+r) U_{k+1}(x)=\frac{(k+r) !}{k !} U_{k+r}(x)$ \\
\hline$w(x, t)=\frac{\partial^{r}}{\partial x^{r}} u(x, t)$ & $W_{k}(x)=\frac{\partial^{r}}{\partial x^{r}} U_{k}(x)$ \\
\hline
\end{tabular}

Where $U_{k}(x), R U_{k}(x), N U_{k}(x)$ and $G_{k}(x)$ are transformed functions of $L u(x, t), R u(x, t), N u(x, t)$ and $g(x, t)$ respectively. Transformed function of initial condition is also as follows:

$$
U_{0}(x)=f(x)
$$

Substituting equation (20) in equation (19) and sweeping an iterative process, finally the values of $U k(x)$ will be determined. Then the inverse transformation of the obtained values of $\left\{U_{k}(x)\right\}_{k=0}^{n}$ will result an approximate solution as follows:

$$
\tilde{u}_{n}(x, t)=\sum_{k=0}^{n} U_{k}(x) t^{k}
$$

Where $n$ is the order of approximate solution. Thus, the exact solution of the problem is as follows:

$$
u(x, t)=\lim _{n \rightarrow \infty} \tilde{u}_{n}(x, t)
$$

\section{VIM Application}

In this section, it is solved Equations (7) and (8) based on VIM. To continue the solution process, heat source term in Equation (7) should be evaluated. One assumption for this term is taken from ${ }^{35}$, i.e. $\psi_{0}=1$ and $\phi(\tilde{t})=(1+\sin (A \tilde{t})) / 2$. To solve Equation (7), the correctional function is written as follows:

$$
\begin{aligned}
& \tilde{T}_{n+1}(\tilde{x}, \tilde{t})=\tilde{T}_{n}(\tilde{x}, \tilde{t})+ \\
& \int_{0}^{\tilde{t}} \lambda_{\tilde{T}}\left[\begin{array}{l}
\frac{\partial \tilde{T}(\tilde{x}, \tau)}{\partial \tau}+V e \frac{\partial \tilde{\tilde{q}}(\tilde{x}, \tau)}{\partial \tilde{x}}+ \\
\frac{1}{N}\left(\beta \tilde{T}^{4}(\tilde{x}, \tau)-\frac{G}{4}\right)-[1+\sin (A \tilde{t})] \exp (-B \tilde{x})
\end{array}\right] d \tau
\end{aligned}
$$

The correctional function of the Equation (8) is of the following form:

$$
\begin{aligned}
& \tilde{q}_{n+1}(\tilde{x}, \tilde{t})=\tilde{q}_{n}(\tilde{x}, \tilde{t})+ \\
& \int_{0}^{\tilde{t}} \lambda_{\tilde{q}}\left[\begin{array}{c}
V e \frac{\partial \tilde{q}_{n}(\tilde{x}, \tau)}{\partial \tau}+\tilde{q}_{n}(\tilde{x}, \tau) \\
+\left[1+\gamma \tilde{\tilde{T}}_{n}(\tilde{x}, \tau)\right] \frac{\partial \tilde{\tilde{T}}_{n}(\tilde{x}, \tau)}{\partial \tilde{x}}
\end{array}\right] d \tau
\end{aligned}
$$

To determine Lagrange multipliers by the new method introduced $^{36}$, we do as follows: we solve the linear part of equations by the Laplace transform method and set it equals to $(-1)^{\mathrm{n}}$, where $n$ is the highest order of the available derivative in the linear part of equation. Boundary conditions appeared in the solution process should be set equal to zero. Finally, everywhere the variable $t$ was appeared, it should be replaced by $(-1)^{n}(\tau-\tilde{t})$. By doing all the aforementioned stages, the Lagrange multipliers for the problem can be obtained:

$$
\begin{aligned}
& \lambda_{\tilde{T}}=-1+\frac{\{1+A(\tilde{t}-\tau)-\cos [A(\tilde{t}-\tau)]\} e^{-B \tilde{x}}}{A}, \\
& \lambda_{\tilde{q}}=-\frac{e^{\frac{\tau-\tilde{t}}{V e}}}{V e} .
\end{aligned}
$$

The only thing remaining is determination of the initial functions. For this purpose, Equation (12) is used with the following initial conditions:

$$
\tilde{T}_{0}(\tilde{x}, \tilde{t})=1, \quad \tilde{q}_{0}(\tilde{x}, \tilde{t})=0 .
$$

This way, VIM formula to determine the desired functions is obtained:

$$
\begin{gathered}
\tilde{T}_{n+1}(\tilde{x}, \tilde{t})=\tilde{T}_{n}(\tilde{x}, \tilde{t})+ \\
\int_{0}^{\tilde{t}} \lambda_{\tilde{T}}\left[\frac{\partial \tilde{T}(\tilde{x}, \tau)}{\partial \tau}+V e \frac{\partial \tilde{q}(\tilde{x}, \tau)}{\partial \tilde{x}}+\right. \\
\left.\frac{1}{N}\left(\beta \tilde{T}^{4}(\tilde{x}, \tau)-\frac{G}{4}\right)-[1+\sin (A \tilde{t})] \exp (-B \tilde{x})\right] d \tau \\
\tilde{q}_{n+1}(\tilde{x}, \tilde{t})=\tilde{q}_{n}(\tilde{x}, \tilde{t})+ \\
\int_{0}^{\tilde{t}} \lambda_{\tilde{q}}\left[\begin{array}{l}
\left.V e \frac{\partial \tilde{q}_{n}(\tilde{x}, \tau)}{\partial \tau}+\tilde{q}_{n}(\tilde{x}, \tau)+\right] \\
\left.\left[1+\gamma \tilde{T}_{n}(\tilde{x}, \tau)\right] \frac{\partial \tilde{T}_{n}(\tilde{x}, \tau)}{\partial \tilde{x}}\right] d \tau
\end{array}\right]
\end{gathered}
$$




\section{RDTM Application}

The transformed form of equation (7) according to iterative equation (19) is as follows:

$$
\begin{aligned}
& V_{k+1}(\tilde{x})=\frac{1}{(k+1)} \times \\
& {\left[\begin{array}{l}
-V e \frac{d U_{k}(\tilde{x})}{d \tilde{x}}-\frac{\beta}{N} \sum_{n=0}^{k} \sum_{l=0}^{n} \sum_{r=0}^{l} V_{r}(\tilde{x}) V_{l-r}(\tilde{x}) V_{n-l}(\tilde{x}) V_{k-n}(\tilde{x}) \\
+e^{-B \tilde{x}}+\frac{1}{k !} e^{-B \tilde{x}} A^{k} \sin \left(\frac{\pi k}{2}\right)
\end{array}\right]}
\end{aligned}
$$

The transformed form of equation (8) based on iterative equation (20) is as follows:

$$
\begin{aligned}
& U_{k+1}(\tilde{x})=\frac{1}{V e(k+1)} \times \\
& {\left[-U_{k}(\tilde{x})-\frac{d V_{k}(\tilde{x})}{d x}-\gamma \sum_{r=0}^{k} V_{k-r}(\tilde{x}) \frac{d V_{r}(\tilde{x})}{d \tilde{x}}\right]}
\end{aligned}
$$

Initial conditions of equation (9) are transformed in the following form:

$$
U_{0}(\tilde{x})=0, \quad V_{0}(\tilde{x})=1 .
$$

By substituting equation (31) in equations (29) and (30), the values of $U_{k}(\bar{x})$ and $V_{k}(\bar{x})$ are obtained. The final solution is also obtained based on equations (21) and (22).

\section{Results and Discussion}

For validating the solution methods, they are compared with ${ }^{31}$ which is a numerical study that is shown in Figure 2. As the figure shows, there are significant differences among the diagram of numerical solution and the diagrams corresponding to VIM and RDTM. Especially the diagram of RDTM doesn't show a proper prediction of the related trend of numerical solution. Quantitatively, the average error for RDTM is $26.42 \%$ and for VIM is $11.56 \%$. Although it seems that the mentioned error is too high for an analytical solution, it should be noted that an analytical solution for a problem similar to the solved problem in the present study (with the same level of nonlinearity) can't be found in the literature. In fact the semi-analytical methods are not able to produce a diagram with such sharp front and if the readers know semi-analytical methods, they will definitely remember the smooth diagrams with soft curves resulted from these methods. Of course, it is necessary to mention that VIM has a less error compared with RDTM and it follows the trend of numerical method somewhat in producing a progressive thermal wave.

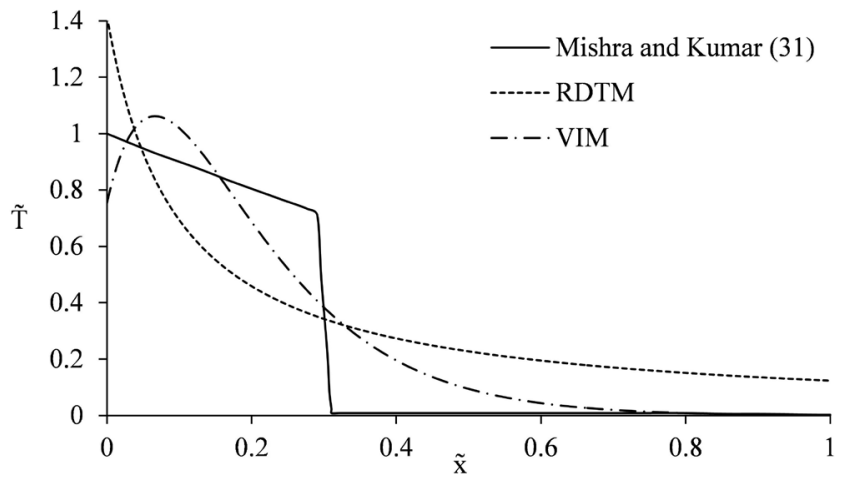

Figure 2. Validation of VIM and RDTM with a Numerical Study (31).

Furthermore, the reason of existence of high errors in boundaries is in fact the lack of imposing the boundary conditions in the process of solving. The linear operator is considered in the direction of dimensionless time, so it is lost the effect of boundary conditions to get to the solution.

An analytical researcher may believe that the mentioned error is so much for solving an analytical solution, but it should be noted that advantages of semi-analytical methods such as VIM compared to the conventional numerical methods make the use of these tools highly desirable for experimental scientists. Determining the approximate solution of the problem helps these researchers to choose their equipment properly and it reduces the cost and the quantity of their experiments.

VIM is used to obtain the rest of results. Figure 3 shows the effect of dimensionless absorption coefficient $(B)$ on the profile of temperature inside the body. As it is seen, with the increase of $B$ in a certain point inside the film, the values of dimensionless temperature decreases, this is natural because according to the equation (7), with the increase of $B$, the dissipation of laser energy becomes steeper and it doesn't have enough time to increase the temperature of the film. Furthermore, with the increase of $\bar{x}$ in a given $B$, the same behavior is seen that is predictable according to equation (6). 


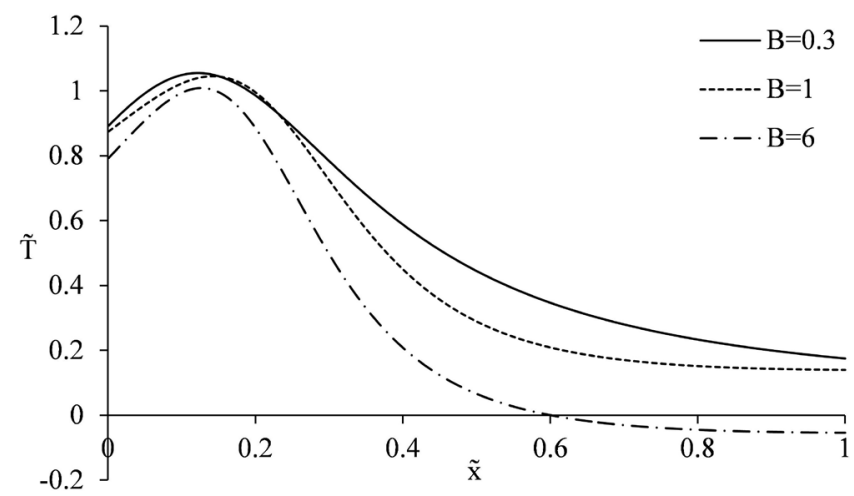

Figure 3. The Effect of B on Temperature Profiles.

The effect of dimensionless time on the temperature profile is shown in Figure 4. With the increase of dimensionless time in a certain point of body, the dimensionless temperature increases. In fact with increasing of time, the thermal energy of laser increases the temperature. Moreover in a given time, the increase of $\tilde{x}$, decreases the dimensionless temperature. Because, with increasing of $\tilde{x}$, the internal energy of the body decreases exponentially and during time, the thermal energy of laser negligibly affects far parts of the body.

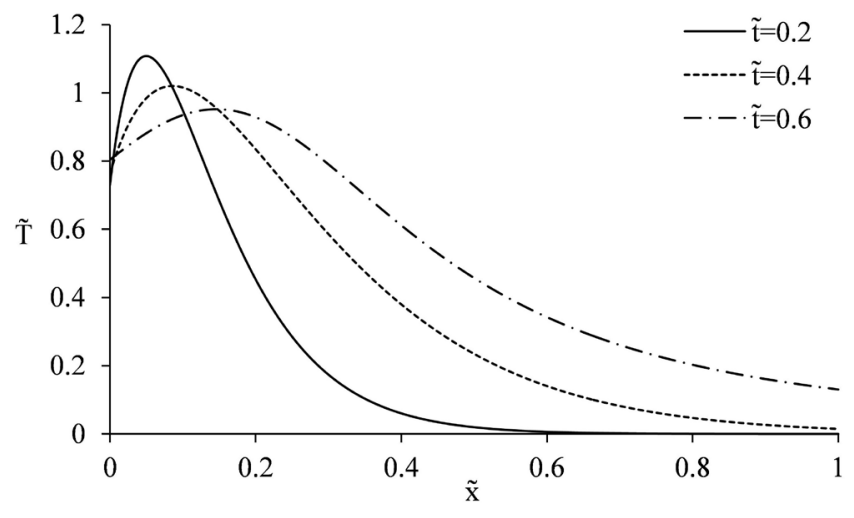

Figure 4. The Effect of $\tilde{t}$ on Temperature Profiles.

In the (Figure 5), the effect of $\gamma$ on the temperature profiles is studied. As it is shown in the figure, increasing of $\gamma$ causes increase of temperature of the body. In some references such $\mathrm{as}^{31}$, a different result has been achieved. In fact these references have reported decreasing of temperature with the increase of $\gamma$. There is an obvious reason for the distinction between the results of present paper and the results of others; different heat source has been chosen. Figure 5 also shows the important effect of variable thermal conductivity. In fact in the sensitive applications that little temperature changes is important, nonlinear analysis has a significant role.

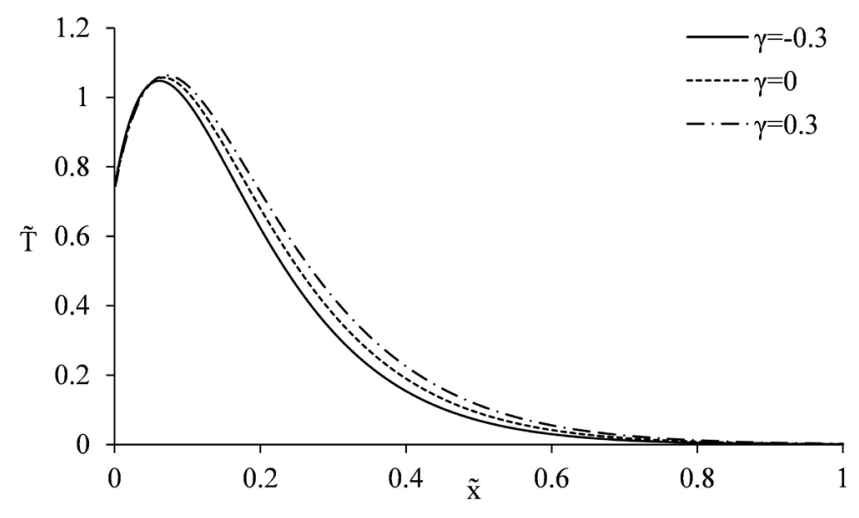

Figure 5. The Effect of $\gamma$ on Temperature Profiles.

The effect of Vernotte number on the temperature profile is seen in the Figure 6. With the increase of Ve, dimensionless temperature also increases. Moreover, the Fourier model $(V e=0)$ shows different behavior compared with non-Fourier model and it predicts lower temperature values. Besides, it is seen that, increase of $V e$ causes temperature variations only up to certain values of $V e$ and beyond of those certain values, variations of $V e$ doesn't have any effect on the variations of temperature.

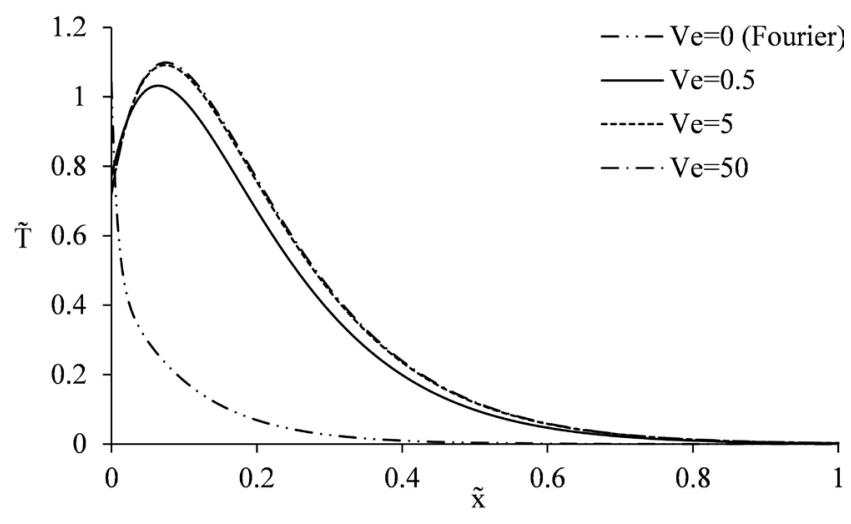

Figure 6. The Effect of Vernotte Number on Temperature Profiles.

\section{Conclusion}

On this paper the effect of laser heating of a metal film temperature was studied. The thermal wave model of Cattaneo-Vernotte was used. Thermal conductivity was 
considered temperature-dependent and the effect of thermal radiation was also considered and then the nonlinear equations were obtained. To solve the equations, semi-analytical methods of RDTM and VIM were used and following results were obtained, briefly:

1) The accuracy of VIM is higher than RDTM.

2) Although VIM has somewhat error, but it can be a good and efficient choice for the researchers who are seeking to determine a relatively good solution of the problem as quick as possible.

3) Considering the thermal conductivity vary with temperature creates a significant difference in the temperature profiles, which shows the importance of nonlinear analysis of the problem.

4) Using laser as a heat source with thermal radiation, remarkable difference between the Fourier and the non-Fourier solutions is evident that must be taken into account in the analysis of similar problems.

5) Only in certain values of relaxation time, temperature variations take place and out of that certain domain, variations of relaxation time doesn't effect on temperature at all.

\section{References}

1. Qiu TQ, Tien CL. Heat Transfer Mechanisms During ShortPulse Laser Heating of Metals. Journal of Heat Transfer. 1993 Nov; 115(4):835-41. Crossref.

2. Raj R, Prasad A, Parida PR, Mishra SC. Analysis of Solidification of a Semitransparent Planar Layer Using the Lattice Boltzmann Method and the Discrete Transfer Method. Numerical Heat Transfer, Part A: Applications. Taylor and Francis. 2006 Feb; 49(3):279-99.

3. Berour N, Lacroix D, Boulet P, Jeandel G. Radiative and conductive heat transfer in a nongrey semitransparent medium. Journal of Quantitative Spectroscopy and Radiative Transfer. 2004 Jun; 86(1):9-30. Crossref.

4. Kim K, Guo Z. Multi-time-scale heat transfer modeling of turbid tissues exposed to short-pulsed irradiations. Computer Methods and Programs in Biomedicine. 2007; 86(2):112-23. Crossref.

5. Kim K, Guo Z. Ultrafast radiation heat transfer in laser tissue welding and soldering. Numerical Heat Transfer, Part A: Applications. Taylor and Francis. 2004 Jul; 46(1):23-40.

6. Catteneo C. A form of heat conduction equation which eliminates the paradox of instantaneous propagation. Compte Rendus. 1958; 247:431-3.
7. Vernotte P. Some possible complications in the phenomenon of thermal conduction. Comptes Rendus de l'Académie des Sciences. 1961; 247:2190-1.

8. Lam TT. Thermal propagation in solids due to surface laser pulsation and oscillation. International Journal of Thermal Sciences. 2010; 49(9):1639-48. Crossref.

9. Al Khairy RT. Thermal wave propagation in a finite medium irradiated by a heat source with Gaussian distribution in both the temporal and spatial domain. International Journal of Thermal Sciences. 2011; 50(8):1369-73. Crossref.

10. Ahmadikia H, Moradi A, Fazlali R, Parsa AB. Analytical solution of non-Fourier and Fourier bioheat transfer analysis during laser irradiation of skin tissue. Journal of Mechanical Science and Technology. 2012; 26(6):1937-47. Crossref.

11. Liu K, Wang J. Analysis of thermal damage to laser irradiated tissue based on the dual-phase-lag model. International Journal of Heat and Mass Transfer. 2014; 70:621-8. Crossref.

12. Liu K. Analysis for high-order effects in thermal lagging to thermal responses in biological tissue. International Journal of Heat and Mass Transfer. 2015; 81:347-54. Crossref.

13. He J. Variational iteration method - a kind of non-linear analytical technique: Some examples. International Journal of Non-Linear Mechanics. 1999; 34(4):699-708. Crossref.

14. Ramos JI. On the variational iteration method and other iterative techniques for nonlinear differential equations. Applied Mathematics and Computation. 2008 May; 199(1):39-69. Crossref.

15. Tatari M, Dehghan M. On the convergence of He's variational iteration method. Journal of Computational and Applied Mathematics. 2007 Oct; 207 (1):121-8. Crossref.

16. Yang S, Xiao A, Su H. Convergence of the variational iteration method for solving multi-order fractional differential equations. Computers and Mathematics with Applications. 2010 Nov; 60(10):2871-9. Crossref.

17. Odibat ZM. A study on the convergence of variational iteration method. Mathematical and Computer Modelling: An International Journal. 2010 May; 51(9-10):1181-92. Crossref.

18. Salkuyeh DK. Convergence of the variational iteration method for solving linear systems of ODEs with constant coefficients. Computers and Mathematics with Applications. 2008 Oct; 56(8):2027-33. Crossref.

19. Saadati R, Dehghan M, Vaezpour SM, Saravi M. The convergence of He's variational iteration method for solving integral equations. Computers and Mathematics with Applications. 2009 Dec; 58(11-12):2167-71. Crossref.

20. Wazwaz A-M. The Variational Iteration Method for Solving New Fourth-Order Emden-Fowler Type Equations. Chemical Engineering Communications. Taylor and Francis. 2015; 202(11):1425-37. Crossref. 
21. Sharma JN, Kaur R. Modeling and analysis of forced vibrations in transversely isotropic thermoelastic thin beams. Meccanica. Springer Netherlands, 2015; 50(1):189-205. Crossref.

22. Hasseine A, Barhoum Z, Attarakih M, Bart H-J. Analytical solutions of the particle breakage equation by the Adomian decomposition and the variational iteration methods. Advanced Powder Technology. 2015; 26:105-12. Crossref.

23. Chen Y, Zhang J, Zhang H. Flapwise bending vibration of rotating tapered beams using variational iteration method. Journal of Vibration and Control. 2014; 22(15):3384-95. Crossref.

24. Keskin Y, Oturang G. Reduced Differential Transform Method for Partial Differential Equations. International Journal of Nonlinear Sciences and Numerical Simulation. 2009; 10(6):741-9. Crossref.

25. Keskin Y, Oturanc G. Reduced differential transform method for solving linear and nonlinear wave equations. Iranian Journal of Science and Technology. 2010; 34(2):113-22.

26. Abazari R, Adem KJJ. Numerical Study of Two-Dimensional Volterra Integral Equations by RDTM and Comparison with DTM. Abstract and Applied Analysis. 2013; 2013: $1-10$.

27. Benhammouda B, Vazquez-Leal H, AS-R. Modified Reduced Differential Transform Method for Partial Differential-Algebraic Equations. Journal of Applied Mathematics. 2014; 2014:1-9.

28. Club YR, Branch CT. Assessment of homotopy perturbation method in non-linear convective-radiative non-fourier conduction. Thermal Science. 2011; 15(2):263-74.

29. Saedodin S, Yaghoobi H, Torabi M. Application of the Variational Iteration Method to Nonlinear Non-Fourier
Conduction Heat Transfer Equation with Variable coefficient. Heat Transfer-Asian Research. 2011; 40(6):513-23. Crossref.

30. Torabi M, Yaghoobi H. Thermal Analysis of Non-linear Convective - Radiative Hyperbolic Lumped Systems with Simultaneous Variation of Temperature-Dependent Specific Heat and Surface Emissivity by MsDTM and BPES. International Journal of Thermophysics. 2013; 34(1):122-38. Crossref.

31. Mishra SC, Kumar TBP. Analysis of a Hyperbolic Heat Conduction-Radiation Problem With Temperature Dependent Thermal Conductivity. Journal of Heat Transfer. 2014; 131(11): 1-7.

32. Malekzadeh $P$, Rahideh H. IDQ two-dimensional nonlinear transient heat transfer analysis of variable section annular fins. Energy Conversion and Management. 2007 Jan; 48(1):269-76. Crossref.

33. Blackwell BF. Temperature Profile in Semi-infinite Body with Exponential Source and Convective Boundary Condition. Journal of Heat Transfer. 1990 Aug; 112(3): 567-71. Crossref.

34. Zubair SM, Chaudhry MA. Heat conduction in a semiinfinite solid due to time-dependent laser source. International Journal of Heat and Mass Transfer. 1996 Sep; 39(14):3067-74. Crossref.

35. Torii S. Heat transfer mechanisms in thin film with laser heat source. International Journal of Heat and Mass Transfer. 2005; 48(3-4): 537-44. Crossref.

36. Samaee SS, Yazdanpanah O, Ganji DD. New approaches to identification of the Lagrange multiplier in the variational iteration method. Journal of the Brazilian Society of Mechanical Sciences and Engineering. 2014; 37(3):937-44. Crossref. 